Wibke Hellmich

Christoph Pelargus

Kai Leffhalm

Alexandra Ros

Dario Anselmetti

Experimental Biophysics and Applied Nanosciences, Physics Department, Bielefeld University, Bielefeld, Germany

\section{Single cell manipulation, analytics, and label-free protein detection in microfluidic devices for systems nanobiology}

\begin{abstract}
Single cell analytics for proteomic analysis is considered a key method in the framework of systems nanobiology which allows a novel proteomics without being subjected to ensemble-averaging, cell-cycle, or cell-population effects. We are currently developing a single cell analytical method for protein fingerprinting combining a structured microfluidic device with latest optical laser technology for single cell manipulation (trapping and steering), free-solution electrophoretical protein separation, and (label-free) protein detection. In this paper we report on first results of this novel analytical device focusing on three main issues. First, single biological cells were trapped, injected, steered, and deposited by means of optical tweezers in a poly(dimethylsiloxane) microfluidic device and consecutively lysed with SDS at a predefined position. Second, separation and detection of fluorescent dyes, amino acids, and proteins were achieved with LIF detection in the visible (VIS) $(488 \mathrm{~nm})$ as well as in the deep UV $(266 \mathrm{~nm})$ spectral range for label-free, native protein detection. Minute concentrations of $100 \mathrm{fm}$ injected fluorescein could be detected in the VIS and a first protein separation and label-free detection could be achieved in the UV spectral range. Third, first analytical experiments with single Sf9 insect cells (Spodoptera frugiperda) in a tailored microfluidic device exhibiting distinct electropherograms of a green fluorescent protein-construct proved the validity of the concept. Thus, the presented microfluidic concept allows novel and fascinating single cell experiments for systems nanobiology in the future.
\end{abstract}

Keywords: Microfluidic device; Miniaturization; Native UV-LIF detection; Protein; Single cell analytics

DOI 10.1002/elps.200500185

\section{Introduction}

In systems biology [1], a multitude of different disciplines from biology, chemistry, physics, material science, microand nanoengineering, and (bio)informatics aim to link quantitative molecular structural and functional (proteomic) information to the different genetically programmed and regulated networks in a living cellular organism. To date, proteomes are analyzed at the level of $10^{5}-10^{6}$ cells accessing functional information only on the basis of that probed cellular ensemble. Averaging effects from cellcycle-dependent states, the different and inhomogeneous cellular response to an external stimulus, or the introduction of genomic and proteomic variabilities during

Correspondence: Dr. Alexandra Ros, Experimental Biophysics \& Applied Nanosciences, Bielefeld University, Universitätsstrasse 25, D-33615 Bielefeld, Germany

E-mail: alexandra.ros@physik.uni-bielefeld.de Fax: +49-521-1062959

Abbreviations: GFP, green fluorescent protein; OT, optical tweezer; PDMS, poly(dimethylsiloxane); Sf, Spodoptera frugiperda; VIS, visible eucaryotic cell proliferation are completely neglected. The analysis of smallest analyte quantities and the hunt for low-abundant proteins at the single cell level, however, requires new techniques for efficient and sensitive separation, detection, and analysis. As a rule of thumb, the typical protein content of a cell is about $15 \%$ proteins w/w which equals $75 \mathrm{pg}, 2 \mathrm{fmol}$, or $10^{8}$ protein molecules, assuming an average molecular mass of $40 \mathrm{kDa}$. A lowabundant protein in a cell at $\mu \mathrm{m}$ concentration runs at the amol level which is equivalent to $10^{5}$ molecules.

In systems nanobiology [2], microfabrication and nanotechnology offer novel tools to detect, measure, analyze, steer, and manipulate individual molecules and cells. Such tools allow more detailed insights into the interplay of genomic information and functional peculiarity at the single molecule or single cell level. Micro total analysis systems ( $\mu$ TAS) or lab-on-a-chip systems $[3,4]$ offer the possibility to handle minute volumes down to the $\mathrm{pL}$ and even $\mathrm{fL}$ range. In recent years, protein electrophoresis, one of the most efficient techniques for protein separation, has been investigated in microfluidic systems [3]. Standard capillary separation techniques were transferred to the 
microchip format and LIF detection systems for the ultrasensitive detection of labeled proteins were developed. Using LIF detection in the visible (VIS) range, separation of covalently labeled proteins $[5,6]$ and peptides $[7,8]$ as well as postcolumn labeling for protein chip electrophoresis have been demonstrated [9, 10]. Recently, a label-free interferometric backscatter method has been described by Wang et al. [11], where proteins could be detected in the nu range. It is worth noting that the achievable detection limit in microfluidic devices upon injecting $\mathrm{nL}$ volumes at $\mathrm{nM}$ concentrations lies in the amol range.

In contrast to microfluidic systems, native LIF detection, based on the fluorescence of the aromatic amino acids tryptophan (Trp), tyrosine (Tyr), and phenylalanine (Phe), has found widespread use in conventional CE. In 1992, Yeung and co-workers [12] pioneered a LIF detection method with $275 \mathrm{~nm}$ excitation light provided by an $\mathrm{Ar}^{+}$laser with pm detection limits. Since then this method has served for the exocytose monitoring of single mast cells [13] as well as for the separation of hemoglobin variants in red blood cells [14]. Exploiting alternative laser systems, nM detection limits for Trp could be achieved with a metal vapor laser [15] or an excimer laser [16]. With solid state lasers, nM detection limits of peptides [17] and proteins $[18,19]$, as well as a pm detection limit for carbonic anhydrase [19] were reported. In contrast to these conventional CE methods protein separation with native UV detection on a quartz microchip with $\mu \mathrm{m}$ detection limit has only been demonstrated in the very recent past [20]. CE could thus be a method of choice for label-free single cell analysis.

First single cell fingerprinting with capillary sieving electrophoresis in 1-D [21, 22] and 2-D format using a protein stain with LIF detection in the VIS range was pioneered by the group of Dovichi and co-workers [23]. Recently, microfluidic devices have been explored for separation and detection of fluorescent dyes [24, 25] and a specific small peptide [26] or vitamin [27] from single cells.

In this work, we focus on three main issues: (i) we describe the trapping, steering, and deposition of a single target cell out of a cell culture by means of optical tweezers (OTs) and consecutive lysis in a microfluidic device. (ii) The separation and detection of native amino acids and proteins by VIS and UV-LIF in a microfluidic device is demonstrated. (iii) The monitoring of a first single cell electropherogram of a fluorescent protein (a green fluorescent protein (GFP)-construct) in a microfluidic device is presented. The manipulation and lysis of single cells and the incorporation of a detection system are based on a poly(dimethylsiloxane) (PDMS) microfluidic chip adapted to an inverted microscope with tailored UV optics and single photoncounting detection. Such microfluidic devices will be a future tool for single cell proteomic fingerprinting.

\section{Materials and methods}

\subsection{Chemicals and reagents}

PDMS (Sylgard 184) was purchased from Dow Corning (Midland, MI, USA). Quartz slides were from SPI Supplies (USA), glass microscope slides from Menzel (Germany). Avidin and Pullulan were obtained from Sigma (Deisenhofen, Germany) and lysocyme C from Serva (Heidelberg, Germany). Trp, PBS tablets, CHES, and Tris were purchased from Fluka (Buchs, Switzerland). Triblock copolymer Pluronics F-108 was a generous gift from BASF (Ludwigshafen, Germany). SDS was from Merck (Darmstadt, Germany). For all solutions deionized water from a Milli-Q biocel (Millipore, Bedford, MA, USA) was used.

\subsection{Cells}

Sf9 insect cells (Spodoptera frugiperda) from Novagen (Madison, WI, USA) transfected with pIEx4-vector (Novagen) containing the gene for the fusion protein were used in these studies. The GFP-Sf9 cells expressed a GFPlabeled "loss-of-function" mutant of the cytoplasmic Gprotein ArF1 of Medicago truncatula. Transfection of cells resulted in maximal 50\% efficiency for live cells containing the GFP-construct protein (T31N-GFP, molecular mass $49.5 \mathrm{kDa}, \mathrm{p} / 5.6)$. Portions of this cell culture $(200 \mu \mathrm{L})$ in BacVector insect cell medium (Novagen) were washed with PBS-buffer (10 mm phosphate, $137 \mathrm{~mm} \mathrm{NaCl}$, $2.7 \mathrm{~mm} \mathrm{KCl}$, titrated to the optimal cell medium $\mathrm{pH}$ 6.4) via centrifugation and subsequent buffer addition. An estimated concentration of $10^{5}$ cells $/ \mathrm{mL}$ was used for the single cell manipulation and lysis experiments.

\subsection{Fabrication of the PDMS device}

A master with the inverted relief of the microstructure was fabricated via spincoating a photoresist (SU-8) onto an Siwafer, UV-exposing through a chromium mask, and developing in a developer bath. The detailed fabrication procedure was recently published by Duong et al. [28]. The polymer Sylgard 184 and its curing agent were mixed in a 10:1 ratio and poured over the microstructured wafer. After curing at $85^{\circ} \mathrm{C}$ for $4.5 \mathrm{~h}$ the cross-linked polymer was easily peeled off the wafer and the reservoir holes were punched through the structured side. The PDMS slab and a clean quartz (for UV) or glass (for VIS detection) slide were oxidized in a UVO-Cleaner (Model 42-220, Jelight, 
USA) for 3 min. Afterwards, the PDMS slab was placed onto the quartz or glass slide forming an irreversible seal. The microfluidic channels had a typical cross section of $20 \times 20 \mu \mathrm{m}^{2}$ and channel walls were coated with a triblock copolymer F-108 [25] significantly reducing unwanted cell adhesion during cell steering.

\subsection{OTs and microfluidic liquid handling}

Individual Sf9 cells were trapped, injected, and steered in the microfluidic channel by a home-made single-beam OT system. The OT was incorporated into an inverted optical microscope [29] with additional standard fluorescence microscopy capabilities in the VIS range. A selfconstructed $x / y$-stage allowed long range positioning of cells with the OTs within the microchannel for maximal $25 \mathrm{~mm}$ with a precision of $1-2 \mu \mathrm{m}$. By this means, an individual cell can be captured in the reservoir and navigated to the injection cross. Cell movement and lysis was observed using a CCD camera (DMK 3002-IR/C, The Imaging Source, Germany) grabbing images at a rate of 25 frames/s.

\subsection{LIF detection in the VIS spectral range}

The experimental setup was mounted on an inverted microscope (Axiovert 100, Zeiss, Jena, Germany), which additionally served as a platform for the confocal LIF detection system (see Fig. 2). Laser light $(488 \mathrm{~nm})$ from an $\mathrm{Ar}^{+}$-ion laser $(25 \mathrm{~mW}$, Omnichrome, Germany or $2 \mathrm{~mW}$, Spectraphysics, Germany) was coupled into the rear port of the microscope via two mirrors (New Focus, USA). The excitation light was reflected by a dichroic mirror and focused by a $20 \times$ objective (Zeiss) into the microchannel. The detection window was adjusted with the $x / y$-stage along the separation channel at various distances from the injector (usually several $\mathrm{mm}$ ). The emitted fluorescence light was collected by the objective and passed through the dichroic mirror and a longpass filter $(520 \mathrm{~nm})$. The tube lens focused the emission light through an $x / y$ adjustable $400 \mu \mathrm{m}$ wide pinhole (unless otherwise stated) onto the photoncounting photomultiplier (Hamamatsu H6240, Japan).

\subsection{LIF detection in the UV spectral range}

For sensitive detection in the UV range, excitation light from a frequency quadrupled $\mathrm{Nd}$ :YAG laser $(266 \mathrm{~nm}$, Nanolase, France) was coupled into the rear port of the microscope (see Fig. 2). The wavelength was adjusted to the autofluorescence excitation maximum of Trp. Switching between the two excitation modes was achieved via a flipper equipped with a silver-coated mirror (New Focus).
For UV excitation and detection, a dichroic mirror (Laseroptik, Germany) with high reflectivity at $266 \mathrm{~nm}$ and high transmission $>300 \mathrm{~nm}$ passed the excitation light through the $52 \times$ reflective objective (Ealing, USA), which focused the laser light on the microchannel. Emitted fluorescence was collected with the reflective objective and focused with a high UV transmission tube lens (Zeiss) through the pinhole onto the photomultiplier. Fluorescence emission spectra of PDMS excited at $266 \mathrm{~nm}$ demonstrated a reduction to approximately $30 \%$ from maximal PDMS background fluorescence for wavelengths above $325 \mathrm{~nm}$ (data not shown). An interference emission filter with high transmission at $360 \mathrm{~nm}(50 \%)$ was thus chosen (360/50, Analysentechnik, Germany).

\subsection{Chip operations}

Initial filling of the microchannels was performed by pipetting the buffer into one reservoir and by applying a vacuum to the other reservoirs. Subsequent flows were either initialized by hydrostatic pressure due to different droplet volumes on the corresponding reservoirs or through electrokinetic pumping. Electrical connection to the microchip device was achieved with four platinum electrodes which were dipped into the reservoirs. Voltage was applied using power supplies from FUG (Modell HCN 14-12500 and HCN 7E-12500, Germany). Instrumental control and data acquisition were performed with software programmed in LabView (National Instruments, Austin, TX, USA). For protein or dye analysis from reservoir 1 the floating method was used according to [30]. Separation buffer for VIS-LIF and UV-LIF separation and detection was Tris-buffer (10 mM, pH 8.2). The single cell lysate was injected into the separation channel 2 by applying a positive voltage to reservoir 4 filled with the separation buffer (100 mm Tris, $100 \mathrm{~mm}$ CHES, 8\% Pullulan, $0.1 \%$ SDS, $\mathrm{pH} 8.6)$.

\section{Results and discussion}

\subsection{Single cell trapping, steering, and lysis}

Diluted insect cells in PBS buffer were pipetted into the buffer reservoir of channel 1 of our microfluidic device (Fig. 1a) where an individual cell was optically selected, trapped, and injected into the microfluidic channel with our OT setup. The injection and steering of the cell (Fig. 1c) was realized with the dedicated $x / y$-stage. The individual cells were transferred to the crossing of our microfluidic device which was microstructured by vertical posts in order to act as a physical cell trap (Figs. 1b and d). Once 

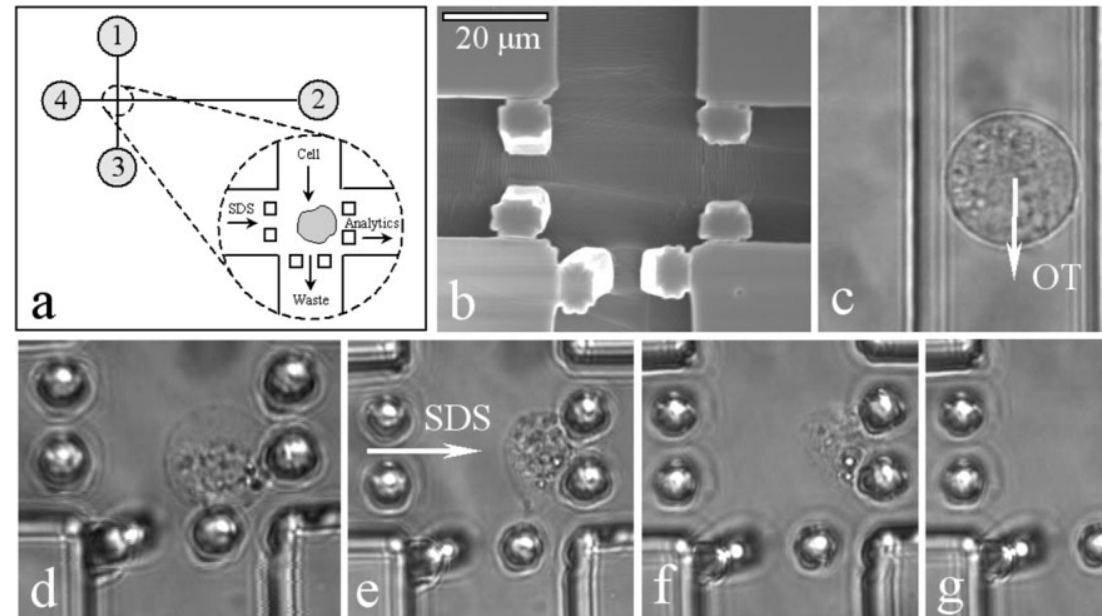

the cell was navigated into this position the optical trap was switched off and the cell was allowed to adhere to the microchannel wall. Consecutively, cell lysis was performed by flushing a $0.5 \%$ SDS solution in PBS by hydrostatic pressure into the perpendicular channel (from channel 4 to 2). The cell lysis was visualized and controlled by optical bright-field microscopy. Figures $1 \mathrm{~d}-\mathrm{g}$ demonstrate a sequence of snapshots from a single cell lysis at the injection point close to the entrance of channel 2 , in which subsequent analytics will be performed. Complete cell lysis was typically achieved within $6 \mathrm{~s}$.

\subsection{LIF setup and VIS-LIF detection}

The detection system for VIS and native UV LIF of proteins, which will be applied to the lysed cells in the future, is schematically depicted in Fig. 2. It is based on an inverted microscope providing a robust and versatile setup for microchip inspection, especially for the optimization of microchip injection and the placement of the detection window in the microchannel. The microfluidic device is constructed as PDMS/glass or PDMS/quartz hybrid in order to minimize fluorescence in the chosen wavelength range. Molded PDMS microchips were thus sealed with a quartz or glass objective slide after oxidative UV treatment of the two components. This UV treatment provided a tight and irreversible seal of the PDMS device both for glass and quartz slides, which was also reported by plasma treatment of PDMS and glass surfaces [31]. The microscope slides served thus as the bottom of the microstructure through which the excitation light passed the microchip.

In order to estimate the sensitivity limit in our microchips, the floating method [32, 33] was chosen to thoroughly study fluorescent dye detection in the VIS range. Thus, microscope.
Figure 1. (a) Scheme of our PDMS microfluidic device. Inset: channel crossing with the cell trap composed of microstructured obstacles, (b) scanning electron micrograph of the cell trap, (c) single cell in a channel navigated by OTs in the microchannel, and $(\mathrm{d}-\mathrm{g})$ optical micrographs of a single cell at the injection position during SDS lysis. SDS flow is from channel 4 through the cell trap into channel 2.

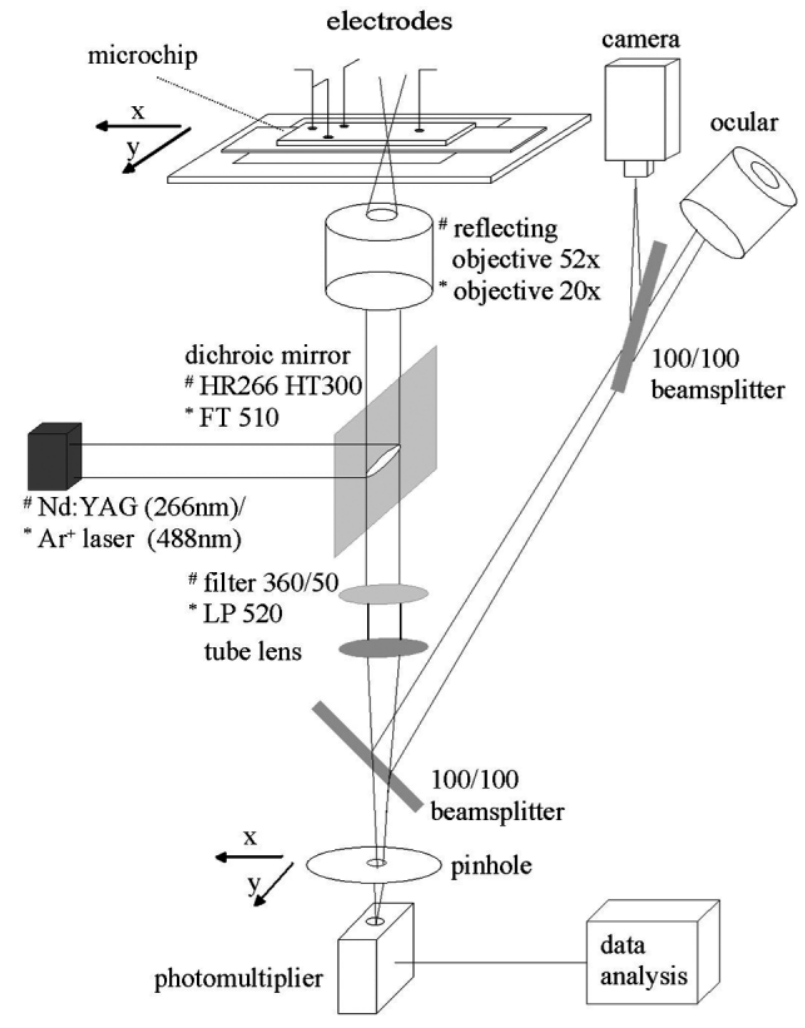

Figure 2. Scheme of the setup for the UV (\#) and VIS $\left(^{*}\right)$ LIF detection systems realized on an inverted optical

extremely small analyte amounts (fM range) could be detected in our PDMS microfluidic devices [30]. Figure 3a shows the electropherogram of an electrokinetically injected $100 \mathrm{fm}$ fluorescein solution. With respect of the detection volume (defined by the pinhole and the microchannel dimensions) this corresponds roughly to $50-100$ molecules. This is well below the anticipated number of 

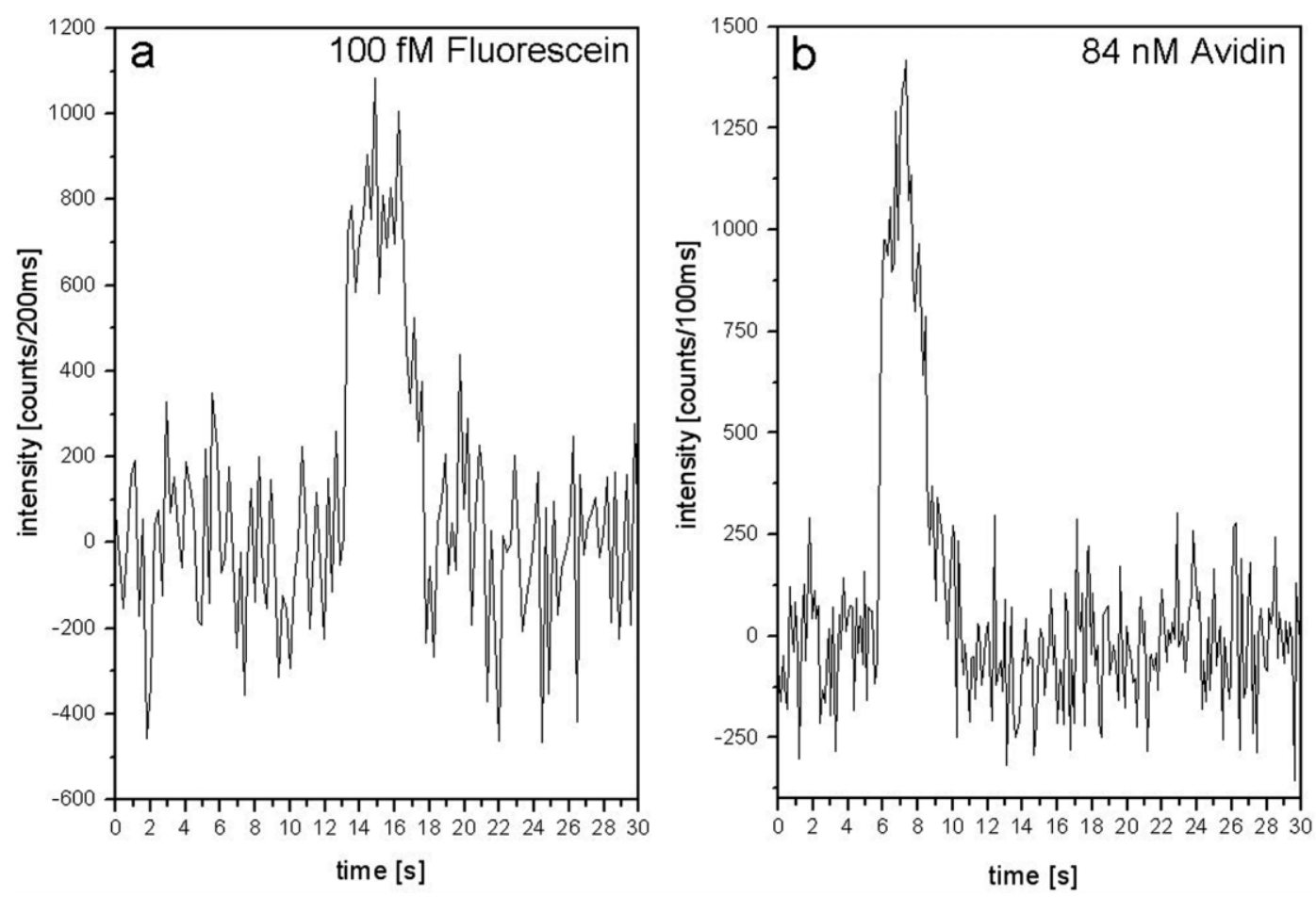

Figure 3. (a) Electropherogram for the VIS LIF detection of $100 \mathrm{fM}$ fluorescein injected into the PDMS microchannel device (separation electric field $1250 \mathrm{~V} / \mathrm{cm}$ ). Baseline correction and background substraction were applied to the original data. (b) Electropherogram for the VIS LIF detection of $84 \mathrm{~nm}$ fluorescein-avidin injected into a PDMS device with a separation field strength of $1500 \mathrm{~V} / \mathrm{cm}$. Laser power was $25 \mathrm{~mW}$ for both electropherograms.

low-abundant protein copies in a single cell. Furthermore this is, to our knowledge, the smallest dye concentration which was electrokinetically injected and detected in a microfluidic device. For the fluorescently labeled protein avidin, an electropherogram resulting from the injection of $84 \mathrm{~nm}$ sample concentration is demonstrated in Fig. 3b. The required amol detection sensitivity for low abundant proteins can thus be achieved already with a standard protein in our detection setup in the VIS range.

\subsection{UV-LIF amino acid detection}

The UV fluorescence setup was optimized for the detection of Trp with an excitation and emission maxima at 295 and $353 \mathrm{~nm}$, respectively [34]. With the present fluorescence detection system, fluorescence of the amino acids Phe and Tyr could also be detected. However, a smaller contribution to the overall signal could be expected due to the different emission and excitation maxima and in addition to the smaller quantum yield of Phe [34]. We have thus successively investigated injection and UV-LIF detection of Trp. The inset of Fig. 4a demonstrates a typical electropherogram obtained with the UV detection system for the injection of $50 \mu \mathrm{M}$ Trp in Tris buffer. The detection window was placed at $10 \mathrm{~mm}$ from the cross injector. Furthermore, a plot of peak heights of injected Trp versus concentration is shown in Fig. 4 (see figure legend for detailed separation conditions). The linear regression with a regression factor of $r=0.998$ demonstrates an excellent linearity for Trp concentrations from 50 to $1000 \mu \mathrm{m}$. For the smallest concentration, an $\mathrm{S} / \mathrm{N}$ of 9 has been determined, so that the theoretical detection limit is $17 \mu \mathrm{M}$ for an $\mathrm{S} / \mathrm{N}$ of 3 . Thus, proteins with at least one Trp in their amino acid composition should be detectable with this setup in the low $\mu \mathrm{m}$ range, if fluorescence quenching is absent.

For $50 \mu \mathrm{m}$ injected Trp the number of theoretical plates $(N)$ resulted in 449, corresponding to a height equivalent of a theoretical plate $(H)$ of $22.3 \mu \mathrm{m}$. This is an order of magnitude higher than obtained for fluorescently labeled amino acids [7, 35]. However, it has been reported that the separation efficiency in PDMS devices is often lower compared to glass devices, it can be satisfactory by careful control of separation parameters [7]. Ocvirk et al. [36] report that only with voltage control on all four reservoirs injections for the dye fluorescein with $N>15000$ could be obtained in PDMS. In contrast to this work, we used a positive voltage at the buffer reservoir during 
separation while all other reservoirs were grounded. This implies a pushback flow of analyte to the sample and sample waste reservoir during separation, so that analyte leakage into the separation channel was prevented. This fact is underlined by the high reproducibility of Trp injections with SDs smaller than $5 \%$ for the peak heights (except the $100 \mu \mathrm{m}$ injections with 9.2\%). Furthermore, we expect that peak-broadening could significantly be reduced in the future with a change of the injection method, e.g., pinched injection or narrow sample channel injectors [32]. However, the actual sensitivity of our UVLIF detection system applied to the chosen hybrid quartz/ PDMS microchip device compares well with a previously reported detection of $2 \mu \mathrm{M}$ Trp in a full body quartz microfluidic chip [20]. The outstanding advantage of PDMS as microstructure material is the relatively low production cost compared to quartz microchips and also its versatility. This is of special interest for single cell handling in a microfluidic device, where structured parts or obstacles can easily be incorporated in order to serve as physical barriers for cell trapping.

\subsection{Protein separation and UV-LIF detection}

Two standard proteins, lysocyme C (6 Trp) and avidin (16 Trp), were selected. Figure 4 shows an electropherogram for the separation of a mixture of $125 \mu \mathrm{m}$ avi- din and $500 \mu \mathrm{m}$ lysocyme $\mathrm{C}$. The protein peaks could clearly be identified by a comparison to single injections of the respective protein (data not shown). After a separation distance of only $12 \mathrm{~mm}$ the proteins were separated nearly to baseline resolution.

It is worth noting that protein separation in PDMS microdevices is strongly influenced by the surface properties so that the detection limit of $17 \mu \mathrm{m}$ for Tyr can only be achieved with adequate control of protein-surface interaction. However, lysocyme $C$ and avidin could be separated without further treatment of the PDMS channel surface. A significant improvement of the detection limit should arise by controlling PDMS protein interaction to circumvent unspecific adhesion. For that reason we are currently investigating PDMS surface coatings with poly(oxyethylene) compounds [37].

\subsection{Single cell electropherograms}

In order to prove the general concept of our single cell analytics approach, we monitored the electropherogram of a single Sf9 cell which was modified by a GFP-variant of a cytoplasmatic G-protein T31N-GFP (Fig. 5). The GFP-cell was optically selected in the PBS buffer reservoir from a solution containing less than $30 \%$ live GFPcells and navigated with OT to the microdevice crossing.
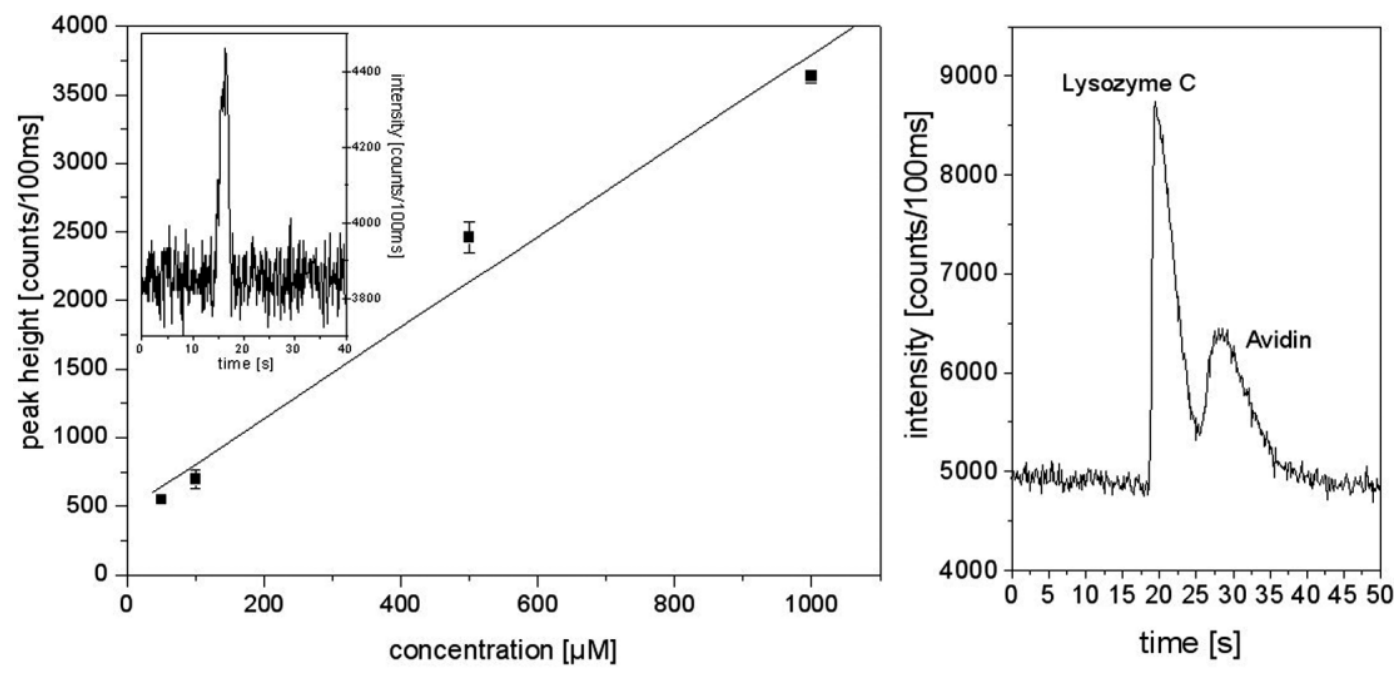

Figure 4. Separation and detection in the UV. (a) Peak heights versus Trp concentration for injections in a PDMS microchannel: the line represents a linear regression with $r=0.998$ indicating a theoretical detection limit of $17 \mu \mathrm{M}$ Trp with $\mathrm{S} / \mathrm{N}=3$. Inset: Electropherogram of $50 \mu \mathrm{m}$ injected Trp in $10 \mathrm{~mm}$ Tris with the detection window placed at $10 \mathrm{~mm}$ after the cross injector. An electrical field of $350 \mathrm{~V} / \mathrm{cm}$ was used during the loading phase of the injection and of $325 \mathrm{~V} / \mathrm{cm}$ during the separation phase, respectively. (b) Electropherogram for the separation of lysocyme $\mathrm{C}$ and avidin. Sample and running buffer was $10 \mathrm{~mm}$ Tris and concentrations of avidin and lysocyme C were 125 and $500 \mu \mathrm{M}$, respectively. For the injection, the analyte was loading the cross injector at $350 \mathrm{~V} / \mathrm{cm}$ and a separation electrical field of $770 \mathrm{~V} / \mathrm{cm}$ was used for separation. Separation length was $12 \mathrm{~mm}$. 


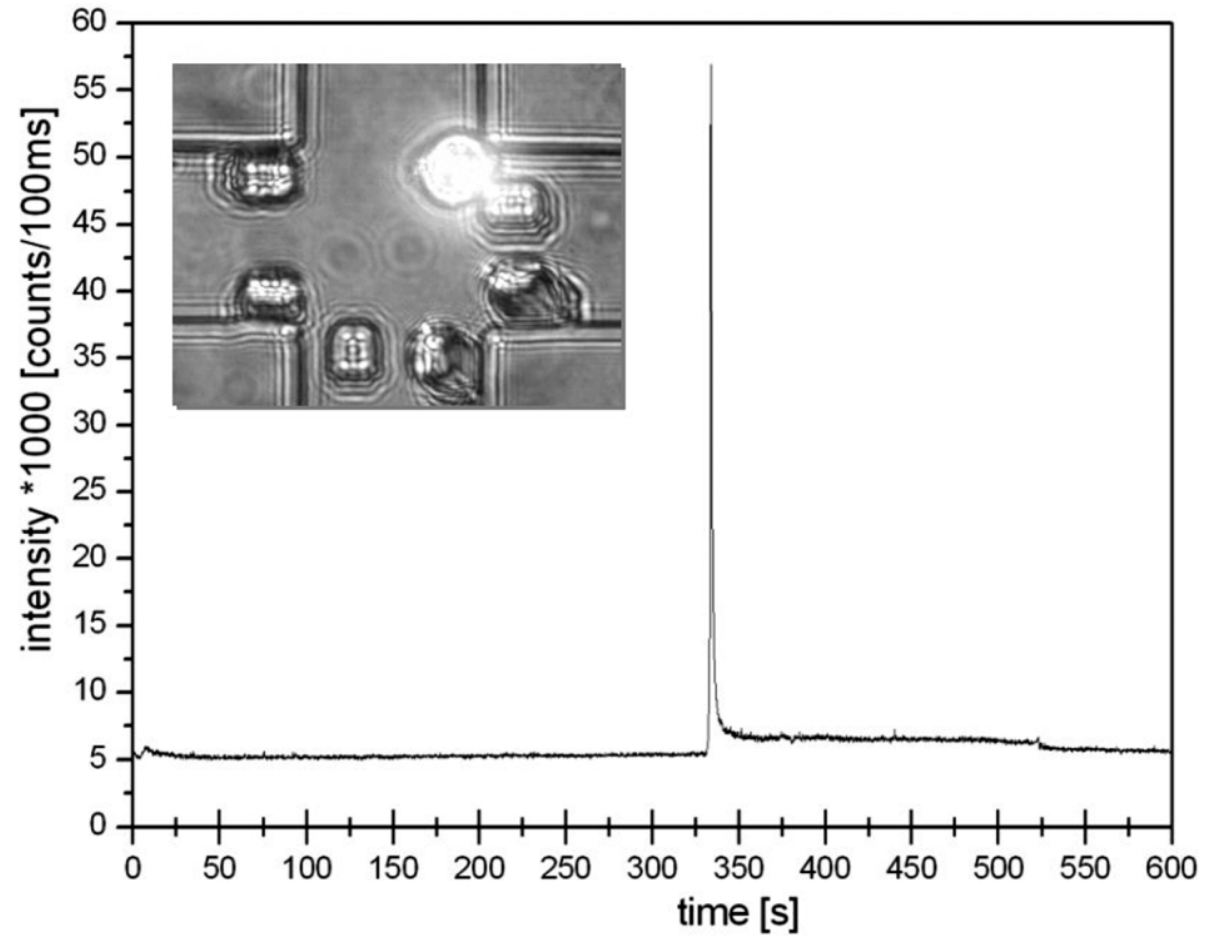

Figure 5. Electropherogram of a single GFP-Sf9 insect cell with a distinct single component peak of the $49.5 \mathrm{kDa}$ T31N-GFP variant. Starting point $(0 \mathrm{~s})$ of the electropherogram is defined by the application of the separation voltage to the horizontal channel and thus does not represent the initiation of cell lysis. Power of the incident laser light (at $488 \mathrm{~nm}$ ) was $2 \mathrm{~mW}$ and the pinhole size $200 \mu \mathrm{m}$. Inset: Fluorescence micrograph of a single GFP-Sf9 insect cell captured at the injection position (for details see text).
There, the OT was switched off and the cell was allowed to adhere (Fig. 5 inset). Subsequently, the separation buffer containing a sieving additive and $0.1 \%$ SDS was electrokinetically injected from reservoir 4 to the captured cell. The electropherogram of the single cell lysate was recorded by VIS LIF detection at a distance of $1000 \mu \mathrm{m}$ from the initial cell position in the microchannel at an electrical field of $100 \mathrm{~V} / \mathrm{cm}$. In the single cell electropherogram (Fig. 5) one distinct peak can be identified at $334 \mathrm{~s}$ with an $\mathrm{S} / \mathrm{N}$ of 400 as expected from a single component analyte, originating from the only fluorescent GFP-construct protein. This peak is characterized by a full width of half maximum of $0.8 \mathrm{~s}$ and a baseline width of only $5 \mathrm{~s}$. This baseline width corresponds well with the lysis time recorded for a cell lysis sequence (Figs. 1d-g) indicating minute diffusion dispersion from the injection process. Thus, the electropherogram denotes transport of the whole lysis fraction to the detection point and reflects the injection of the complete protein content from a single cell cytoplasm. This is to our knowledge the first electropherogram of a single protein compound from a single cell in a microfluidic device.

\section{Concluding remarks}

We are currently developing a microfluidic chip platform which combines single cell trapping, steering, deposition, lysis, and subsequent electrophoretical protein separation, and LIF-detection in the VIS and in the UV spectral range. We have demonstrated the manipulation and controlled lysis of single Sf9 insect cells as well as the separation of proteins with native, label-free UV-LIF detection in a microfluidic PDMS device. Avidin and lysocyme $\mathrm{C}$ were separated with nearly baseline resolution within less than $40 \mathrm{~s}$. Injections of the amino acid Trp resulted in a theoretical detection limit of $17 \mu \mathrm{m}$ which should be also applicable for proteins. We have also demonstrated that our detection setup in the VIS spectral range is capable of detecting minute concentrations necessary for the analysis of lowabundant proteins from a single cell.

In addition, we injected and lysed single Sf9 insect cells expressing a single GFP-construct protein in our microfluidic device and monitored the corresponding single cell electropherogram in the visual spectral range. These first single cell analytical experiments exhibited the distinct feature of a single fluorescent component, in agreement with the probed cell species. Further optimization of the UV optical detection setup will significantly improve separation efficiencies and detection sensitivity, and facilitate a label-free microchip based single cell device for systems nanobiology in the future.

Sf9 cells were generously donated by Nickels Jensen and Professor Karsten Niehaus from the Genetics Department of Bielefeld University. Financial support from the Deutsche Forschungsgemeinschaft (Project: Single Cell Analytics - An 370/1-2) and the generous Pluronics sample donation by BASF (Dr. Stephan Altmann and Dr. Kati 
Schmidt) is gratefully acknowledged. We thank Andy Sischka, Dr. Katja Tönsing, and Thanh Tu Duong from the Experimental Biophysics Group for technical assistance in OT manipulation and microfabrication.

Received March 3, 2005

Revised May 21, 2005

Accepted May 24, 2005

\section{References}

[1] Ideker, T., Galitski, T., Hood, L., Annu. Rev. Genomics Hum. Genet. 2001, 2, 343-372.

[2] Heath, J. R., Phelps, M. E., Hood, L., Mol. Imaging Biol. 2003, 5, 312-325.

[3] Auroux, P. A., lossifidis, D., Reyes, D. R., Manz, A., Anal. Chem. 2002, 74, 2637-2652.

[4] Reyes, D. R., lossifidis, D., Auroux, P. A., Manz, A., Anal. Chem. 2002, 74, 2623-2636.

[5] Liu, Y., Foote, R. S., Culbertson, C. T., Jacobson, K. B., Ramsey, R. S., Ramsey, J. M., J. Microcol. Sep. 2000, 12, 407-411.

[6] Bousse, L., Mouradian, S., Minalla, A., Yee, H., Williams, K., Dubrow, R., Anal. Chem. 2001, 73, 1207-1212.

[7] Lacher, N. A., de Rooij, N. F., Verpoorte, E., Lunte, S. M., J. Chromatogr. A 2003, 1004, 225-235.

[8] Chiem, N., Harrison, D. J., Anal. Chem. 1997, 69, 373-378.

[9] Liu, Y., Foote, R. S., Jacobson, S. C., Ramsey, R. S., Ramsey, J. M., Anal. Chem. 2000, 72, 4608-4613.

[10] Colyer, C. L., Mangru, S. D., Harrison, D. J., J. Chromatogr. A 1997, 781, 271-276.

[11] Wang, Z., Swinney, K., Bornhop, D. J., Electrophoresis 2003, 24, 865-873.

[12] Lee, T. T., Yeung, E. S., J. Chromatogr. 1992, 595, 319-325.

[13] Lillard, S. J., Yeung, E. S., McCloskey, M. A., Anal. Chem. 1996, 68, 2897-2904.

[14] Lillard, S. J., Yeung, E. S., Lautamo, R. M. A., Mao, D. T., J. Chromatogr. A 1995, 718, 397-404.

[15] Zhang, X., Sweedler, J. V., Anal. Chem. 2001, 73, 56205624.

[16] Paquette, D. M., Song, R., Banks, P. R., Waldron, K. C., J. Chromatogr. A 1998, 714, 47-57.
[17] Kuijt, J., van Teylingen, R., Nijbacker, T., Ariese, F., Brinkman, U. A. Th., Gooijer, C., Anal. Chem. 2001, 73, 50265029.

[18] Chan, K. C., Muschik, G. M., Issaq, H. J., Electrophoresis 2000, 21, 2062-2066.

[19] Tseng, W.-L., Chang, H.-T., Anal. Chem. 2000, 72, 48054811.

[20] Schulze, P., Ludwig, M., Kohler, F., Belder, D., Anal. Chem. 2005, 77, 1325-1329.

[21] Hu, S., Zhang, L., Krylow, S., Dovichi, N. J., Anal. Chem. 2003, 75, 3495-3501.

[22] Hu, S., Zhang, L., Newitt, R., Aebersold, R., Kraly, J. R., Jones, M., Dovichi, N. J., Anal. Chem. 2003, 75, 3502-3505.

[23] Hu, S., Michels, D. A., Abu Fazal, M., Ratisoontorn, Ch., Cunningham, M. L., Dovichi, N. J., Anal. Chem. 2004, 76, 4044-4049.

[24] McClain, M. A., Culbertson, C. T., Jacobson, S. C., Albritton, N. L., Sims, C. E., Ramsey, J. M., Anal. Chem. 2003, 75, 5646-5655.

[25] Munce, N. R., Li, J., Herman, P. R., Lilge, L., Anal. Chem. 2004, 76, 4983-4989.

[26] Gao, J., Yin, X. F., Fang, Z.-L., Lab Chip 2004, 4, 47-52.

[27] Xia, F., Jin, W., Yin, X., Fang, Z.-L., J. Chromatogr. A 2005, 1063, 227-233.

[28] Duong, T., Kim, G., Ros, R., Streek, M., Schmid, F., Brugger, J., Ros, A., Anselmetti, D., Microelectr. Eng. 2003, 67-68, 905-912.

[29] Sischka, A., Eckel, R., Toensing, K., Ros, R., Anselmetti, D., Rev. Sci. Instrum. 2003, 74, 4827-4831.

[30] Ros, A., Hellmich, W., Duong, T., Anselmetti, D., J. Biotechnol. 2004, 122, 65-67.

[31] Duffy, D. C., Cooper McDonald, J., Schueller, O. J. A., Whitesides, G. M., Anal. Chem. 1998, 70, 4974-4984.

[32] Zhang, C.-X., Manz, A., Anal. Chem. 2001, 73, 2656-2662.

[33] Effenhauser, C. S., Bruin, G. J. M., Paulus, A., Ehrat, M., Anal. Chem. 1997, 69, 3451-3457.

[34] Lakowicz, J. R., Principles of Fluorescence Spectroscopy, Kluwer Academic/Plenum Publisher, New York 1999.

[35] Seiler, K., Harrison, D. J., Manz, A., Anal. Chem. 1993, 65, 1481-1488.

[36] Ocvirk, G., Munroe, M., Tang, T., Oleschuk, R., Westra, K., Harrison, D. J., Electrophoresis 2000, 21, 107-115.

[37] Hellmich, W., Regtmeier, J., Duong, T., Ros, R., Anselmetti, D., Ros, A., Langmuir 2005, 21, 7551-7557. 